\title{
In-situ TEM Study of Formation of an Ordered Hollow Structure Metalattice from Silica Nano-Opals through High-Temperature Annealing
}

\author{
Parivash Moradifar ${ }^{1}$, Jennifer L. Russell ${ }^{2}$, Thomas E. Mallouk ${ }^{2}$ and Nasim Alem ${ }^{1}$ \\ ${ }^{1 .}$ Department of Materials Science and Engineering, Materials Research Institute, The Pennsylvania \\ State University, University Park, PA. \\ 2. Department of Chemistry, The Pennsylvania State University, University Park, PA.
}

Metalattices are artificial 3D ordered arrays of nanostructured solids with modulated electronic, optical, magnetic, and vibrational degrees of freedom with highly interconnected surface or interface on periodicity range between $1 \mathrm{~nm}-100 \mathrm{~nm} .{ }^{[1,2,3]}$ On the other hand, hollow nanostructures, with large surface area, low density (large interior/exterior surface area), and high chemical/thermal stability have attracted great attention in many fundamental research areas and practical applications ranging from controlled drug delivery, photonics, as well as gas and heavy ion adsorbent membranes. In particular, hollow silica spheres have been proposed as promising candidates for photonic crystals and antireflection films due to Mie resonance and great mechanical properties. ${ }^{[4]}$

This study will present controlled formation of ordered hollow silica spheres under in-situ TEM heating conditions in the temperature range of $\mathrm{RT}$ to $1200{ }^{\circ} \mathrm{C}$ in vacuum. Using in-situ heating scanning/transmission electron microscopy (S/TEM) imaging and spectroscopy, this study explores microstructural transformation and thermal stability of ordered silica nano-opals during post-annealing treatment at elevated temperatures with no chemical agents. ${ }^{[5,6]}$ This investigation also shows the chemical, geometrical, and microstructural transformation that occur in $100 \mathrm{~nm}$ ordered silica nano-opal metalattices.

Hollow-structured silica nano-opals are formed during in-situ TEM post annealing treatment with a high thermal stability. Figure 1 shows a series of TEM images taken at various temperatures. Figures $1 \mathrm{a}-\mathrm{b}$ show no observable change in the morphology of nano-opals before $600{ }^{\circ} \mathrm{C}$ and the structure remains intact retaining its long-range network connectivity. At $900^{\circ} \mathrm{C}$ (Figure 1c), a hollow silica nanostructure starts to form and at $1200^{\circ} \mathrm{C}$, a stable and complete silica nano-opal hollow structure can be observed (Figure 1d). Despite formation of a silica hollow nanostructure, the structure retains its 3-D order with no significant change in stacking or deformation. This study will further explore the changes in the plasmon generation of this $3 \mathrm{D}$ hollow structure resulting from this transformation.

The rearrangement of the silica network and the interfacial contact points between adjacent silica nanoopals are expected to significantly influence the thermal stability, thermal conductivity, and plasmon generation along the network of hollow silica nano-opals. ${ }^{[4]}$ Furthermore, the post-annealing treatment at elevated temperatures (above $900{ }^{\circ} \mathrm{C}$ ), seems to strengthen the covalent linkage and increase the degree of cross-linking at the interfacial contact points of the silica nano-opals. ${ }^{[4]}$

Investigating the unique thermal behavior of silica nano opals and understanding the changes in the 3D ordered structure arising from the formation of a stable ordered hollow structure under post-annealing treatment conditions, can provide insight not only into the high temperature behavior of such 3D ordered metalattice materials, but also on the plasmonic behavior arising from the hollow structured morphology. This understanding is key to develop hollow silica ordered nano-opals as a promising candidate for novel 
insulating materials and high temperature nano-plasmonic applications [7].

References:

[1] Y.Liu et al, Nano Lett. 18(1) p. 546.

[2] J.E.Han and V.H. Crespi, Phyiscal Review Letters 86 (2001), p. 696.

[3] J.E.Han and V.H. Crespi, Phyiscal Review Letters 89 (2002), p. 197203.

[4] D. Zheng, C. Huang and X. Wang, Nanoscale 7 (2015), p. 465.

[5] P. Moradifar et al, Microscopy and Microanalysis 22 (2016), p. 1638.

[6] P. Moradifar et al, Micrsocopy and Micranalysis, 23 (2017), p. 956.

[7] This work was funded by the Penn State MRSEC, Center for Nanoscale Science, under the award NSF DMR-1420620.
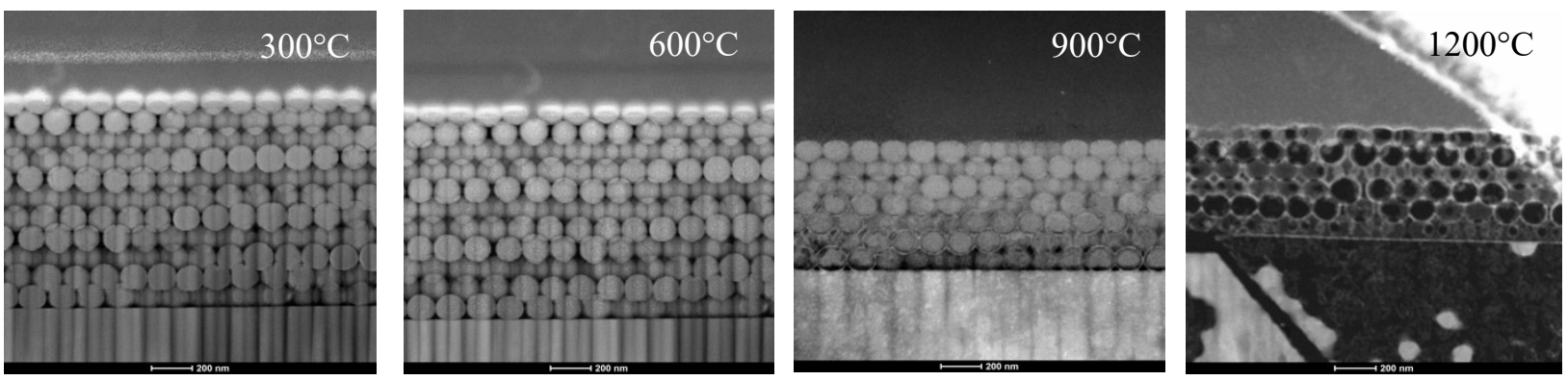

Figure 1. HAADF-STEM image of (a) silica nano-opals at $300^{\circ} \mathrm{C}$ (b) silica nano-opals at $600^{\circ} \mathrm{C}$ (c) silica nano-opals at $900{ }^{\circ} \mathrm{C}$ (d) silica nano-opals at $1200^{\circ} \mathrm{C}$. 